\title{
OSTEOTOMIA VALGIZANTE PARA PSEUDARTROSE DO COLO DO FÊMUR: RELATO DE 32 CASOS
}

\author{
VALGUSING INTERTROCHANTERIC OSTEOTOMY FOR THE TREATMENT \\ OF FEMORAL NECK NON-UNIONS: REPORT OF 32 CASES
}

Carlos Roberto Schwartsmann', Marco Aurélio Telöken², Leonardo Carbonera Boschin², Ivo Schmiedt³, Ricardo Falavinha ${ }^{4}$, Marcus Vinicius Crestani ${ }^{5}$

\section{RESUMO}

Objetivo: Avaliar a consolidação pós-osteotomia osteotomia valgizante do fêmur no tratamento do pseudartrose do colo femoral. Método: Durante 15 anos (1988-2003), 32 casos de pseudartrose do colo do fêmur foram tratados com osteotomia valgizante e fixação. O seguimento médio dos casos foi de 9,8 anos e a média de idade dos pacientes foi de 41,7 anos. Resultados: Dos 32 casos quatro evoluíram para prótese total do quadril e 28 evoluíram para consolidação $(87,4 \%)$. Oito casos evoluíram necrose parcial. Conclusão: A osteotomia valgizante é altamente eficaz para obtenção da consolidação na pseudartrose do colo do fêmur 87,4\% (28/32) A recuperação integral da função do quadril só foi obtida em $56,2 \%(18 / 32)$

Descritores - Fraturas do colo femoral; Osteotomia; Pseudartrose; Relatos de casos

\section{ABSTRACT}

Purpose: The purpose of the present study was to review the results of femoral neck non-unions treatment with valgusing intertrochanteric osteotomy. Methods: Between 1988 and 2003 we treaded thirty two femoral neck non-unions with valgusing osteotomy and fixation. The mean follow-up time was 9.8 years and the mean age was 41.7 years. Results: Twenty eight (87.4\%) of the thirty two valgusing osteotomies evolved to femoral neck union, while four cases (12.6\%) evolved to total hip arthroplasty. Eight cases evolved to partial osteonecrosis. Conclusions: The valgusing intertrochanteric osteotomy for treating femoral neck non-unions achieved consolidation in $87.4 \%$ (28/32). However, only 56.2\% (18/32) achieved full recovery of hip function.

Keywords - Femoral neck fractures; Osteotomies; Pseudoarthrosis.

O objetivo do presente estudo é avaliar a consolidação da osteotomia valgizante na pseudoartrose do colo do fêmur.

\section{MÉTODO}

De janeiro de 1988 até outubro de 2003, no Serviço de Ortopedia e Traumatologia do Complexo Hospitalar Santa Casa de Porto Alegre/RS, foram tratados 32 casos de pseudartrose do colo do fêmur com osteotomia valgizante e fixação, sendo 20 do sexo masculino e 12 do feminino. O paciente mais jovem possuía 18 anos e o mais idoso 66. A média de idade foi de 41,7 anos. $O$ seguimento foi de 9,8 anos (1-15 anos).

Inicialmente as fraturas foram classificadas como:

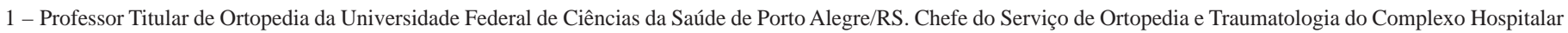
Santa Casa de Porto Alegre/RS.

2 - Médicos do Grupo do Quadril do Serviço de Ortopedia e Traumatologia do Complexo Hospitalar Santa Casa de Porto Alegre/RS.

3 - Médico do Grupo do Joelho do Serviço de Ortopedia e Traumatologia do Complexo Hospitalar Santa Casa de Porto Alegre/RS.

4 - Membro da Sociedade Brasileira de Ortopedia e Traumatologia e do SIRTE. Médico do Hospital Universitário Cajuru.

5 - Médico do Serviço de Ortopedia e Traumatologia do Complexo Hospitalar Santa Casa de Porto Alegre/RS.
} 
Garden I (zero), Garden II (zero), Garden III(14 casos) e Garden IV (18 casos).

Em relação ao tratamento inicial, nove casos chegaram ao hospital sem nenhum tratamento. Os outros 23 casos foram inicialmente fixados com parafusos (13), DHS (7), um com três fios de Steinman e dois com placa angulada laminar (AO).

O tempo médio transcorrido da fratura até a osteotomia, isto é de pseudartrose, foi de 6,5 meses (3,5 - 12 meses).

A decisão de realizar a osteotomia foi baseada apenas no critério radiológico (esfericidade da cabeça). Em apenas três casos foi realizada a ressonância magnética. Em todas haviam sinais de necrose parcial que não foram considerados uma contra-indicação para realização da osteotomia.

A técnica utilizada para osteotomia foi a descrita por Pauwels em $1935^{(1)}$ com ressecção de cunha lateral de $30^{\circ}$ a $50^{\circ}$ e fixação da osteotomia (Figura 1 ).
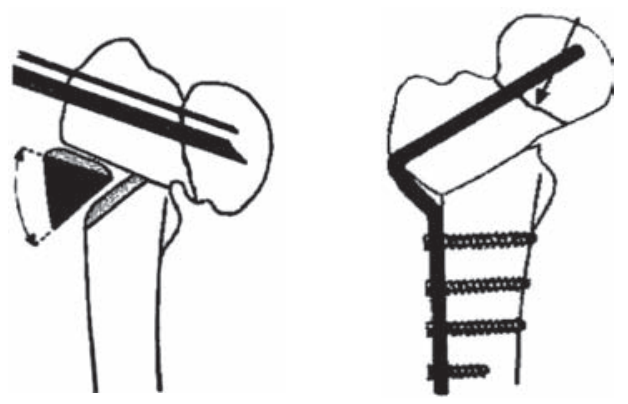

Figura 1 - Técnica de osteotomia intertrocantérica descrita por Pauwels (2).

Após osteotomia, para fixação da mesma, foram utilizados os seguintes materiais: placa de Maclaughlin (01), placa AO (10), placa AO + parafuso antirotatório (04), DCS (01), DHS $150^{\circ}$ (02) e DHS $130^{\circ}$, (14).

Após a osteotomia a ordem de apóio somente era permitida após 90 dias. A partir de então era permitido apoio parcial progressivo com muletas até a consolidação.

As alterações mecânicas promovidas pela osteotomia foram avaliadas e foram mensuradas. A média do momento abdutor do lado da osteotomia foi de 64,8 $\mathrm{mm}$ (54 - $75 \mathrm{~mm})$. O lado contralateral (normal) foi de 73,7 mm (63 - $92 \mathrm{~mm})$.

Portanto em média houve uma diminuição de 8,9mm. Percentualmente houve um decréscimo do momento abdutor de $12,1 \%$.

A média do off-set femoral pós osteotomia foi de 24,9 mm (13 - 36mm). O lado contralateral (normal) foi de 45,5 mm (32 - 58 mm). Portanto em média houve diminuição do off-set femoral de 20,6mm. Percentualmente o decréscimo foi de 45,3\%.
O ângulo cervico-diafisário médio do lado normal foi $131,5^{\circ}\left(120^{\circ}-145^{\circ}\right)$. O ângulo médio pós osteotomia foi de $144,4^{\circ}\left(130^{\circ}-152^{\circ}\right)$. Portanto em média houve valgização de 12,9 mm. Percentualmente a média valgização comparada com o lado normal foi de $9,8 \%$.

\section{RESULTADOS}

Dos 32 casos, quatro pseudartroses evoluíram para prótese total do quadril. 28 osteotomias consolidaram. Em 27 casos foi obtida a consolidação após única osteotomia. Um caso evoluiu para consolidação após nova osteotomia e troca do material de síntese.

As 28 osteotomias consolidadas foram avaliadas de acordo com a classificação de INOUE para necrose avascular. 20 casos foram considerados sem necrose. No estágio dois (densidade anormal ou irregular com discreto achatamento) encontramos cinco casos. No estágio três (densidade irregular com colapso segmentar) encontramos dois casos. Um caso com evolução de 14 anos foi considerado estágio quatro (osteoartrite com deformidade da cabeça), mas até a presente avaliação não foi necessária a realização da artroplastia total do quadril.

Os pacientes foram avaliados pelo método de D’Aubigné et $\mathrm{al}^{(2)}$. Dos 28 casos, 18 foram considerados com 17 e 18 pontos. Os 10 casos restantes obtiveram 15 ou 16 pontos. Nenhum dos pacientes necessitava utilizar qualquer suporte (muleta ou bengala), mas oito apresentavam discreta claudicação no momento da avaliação. Nenhum paciente referiu queixas em relação ao joelho homolateral.

\section{DISCUSSÃO}

A fratura do colo do fêmur já foi descrita como a fratura sem solução ${ }^{(3)}$.

Apesar do avanço da técnica operatória e do desenho e qualidade do material de síntese empregado, a necrose avascular da cabeça femoral e a pseudartrose continuam sendo as principais complicações da fratura.

Apesar da meta do tratamento da pseudartrose ser a de preservar cabeça através da consolidação da fratura, a escolha do tratamento ideal depende basicamente da idade do paciente, da esfericidade e congruência da cabeça femoral, da qualidade do osso existente e da experiência do ortopedista.

O problema além de ser biológico e fundamentalmente biomecânico como demonstrou Pauwels ${ }^{(1)}$. Para o tratamento da pseudartrose ele descreveu uma osteotomia intertrocantérica valgizante que converte as forças de cizalhamento em forças de compressão e obtém a consolidação. 
A artroplastia geralmente é indicada em pacientes acima de 60 anos $^{(4-6)}$, mas a idade fisiológica deve ser mais considerada que a idade cronológica.

Marti et $\mathrm{al}^{(7)}$ indicam a osteotomia valgizante em pacientes até 70 anos.

A presença isolada de necrose avascular sem colapso da cabeça não é contra-indicação para enxertias ou osteotomias. Vários resultados satisfatórios são publicados na literatura ${ }^{(4,5,7-10)}$.

Por isso a simples avaliação radiográfica (esfericidade da cabeça) é o único exame realmente necessário para avaliar a indicação de osteotomia.

A fratura do colo femoral é comum na população idosa, mas a incidência maior de pseudoartrose é mais comumente encontrada nos pacientes mais jovens e que sofreram fraturas por trauma de média e alta intensidade ${ }^{(11-13)}$.

A incidência de pseudartrose na fratura do colo do fêmur ocorre de $10 \%$ a $59 \%$ dos $\operatorname{casos}^{(12-18)}$.

Não existe critério definitivo para concluir se a fratura evoluiu para pseudartrose, mas o tempo mínimo de seis meses deve ser considerado. Barnes et $\mathrm{al}^{(14)} \mathrm{em}$ estudo prospectivo multicêntrico concluiu que apenas $14 \%$ das fraturas intracapsulares deslocadas consolidaram em seis meses.

A história clínica é muito importante: dor persistente na virilha, nádega, anterior da coxa ou joelho que é exacerbada por movimentos rotacionais devem ser considerados casos suspeitos e bem acompanhados.

Radiografias seriadas geralmente são necessárias para se chegar ao diagnóstico. Alho et $\mathrm{al}^{(18)}$, revisando 203 casos, descreveram fatores preditivos da não união: mudança de posição dos parafusos, mudança do traço da fratura e extrusão de parafusos maior que $20 \mathrm{~mm}$.

Na presente série nove pacientes chegaram ao hospital sem nenhum tratamento prévio e, portanto, o diagnóstico foi evidente, mas a média de tempo até o diagnóstico final foi de 6,5 meses.

O critério radiológico foi o único utilizado em todos os casos para indicar a osteotomia.

Apesar da maioria dos autores utilizarem a placa la$\operatorname{minar}^{(4,5,7,19)}$ para fixação da osteotomia na metade dos nossos casos como foi utilizado o parafuso deslizante DHS (16/32) (Figura 2).

O parafuso deslizante (DHS) é menos agressivo, permite compressão no foco de pseudoartrose e sua execução é tecnicamente mais fácil.

Em nenhum caso foi colocado enxerto ósseo no foco de pseudartrose.

Mathews et $\mathrm{al}^{(5)}$ foram os primeiros a mensurar as alterações mecânicas que ocorrem após a osteotomia.
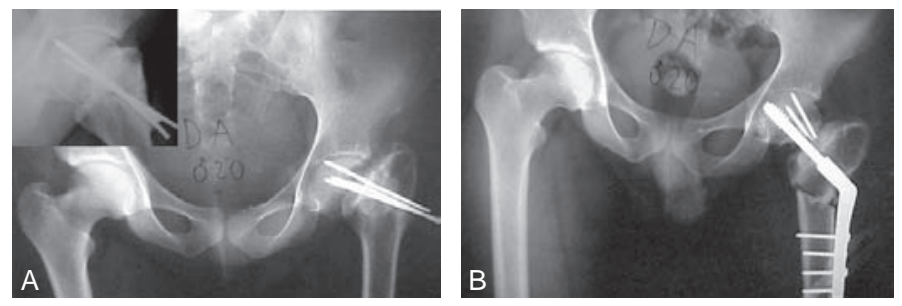

Figura 2 A - D.A., 20 anos masc.: Rx inicial. Pseudartrose do colo do fêmur $\mathrm{E}$. Osteossíntese com três fios de Steinmann há oito meses. B - Rx panorâmico de bacia após osteotomia valgizante. Não foi possível retirar dois fios de Steinmann. Osteosintese com DHS.
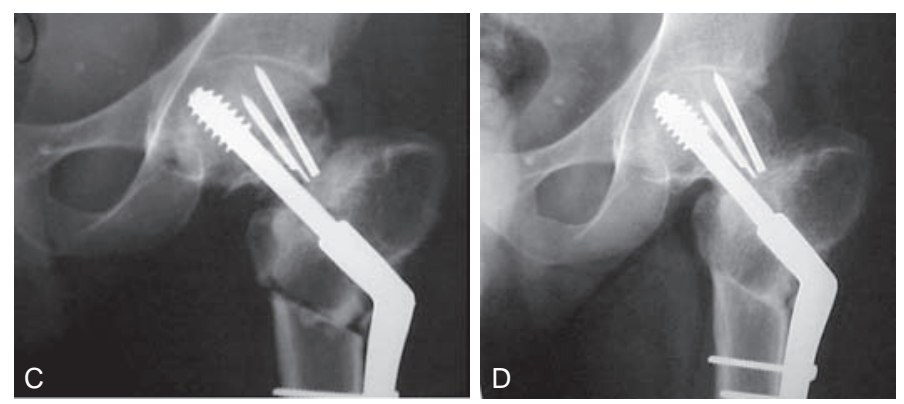

Figura $2 \mathrm{C}-\mathrm{Rx}$ em detalhe do quadril E no pós-operatório imediato. D - Osteotomia e pseudartrose consolidada. Observar fio de Steinmann superior que está subcondral.
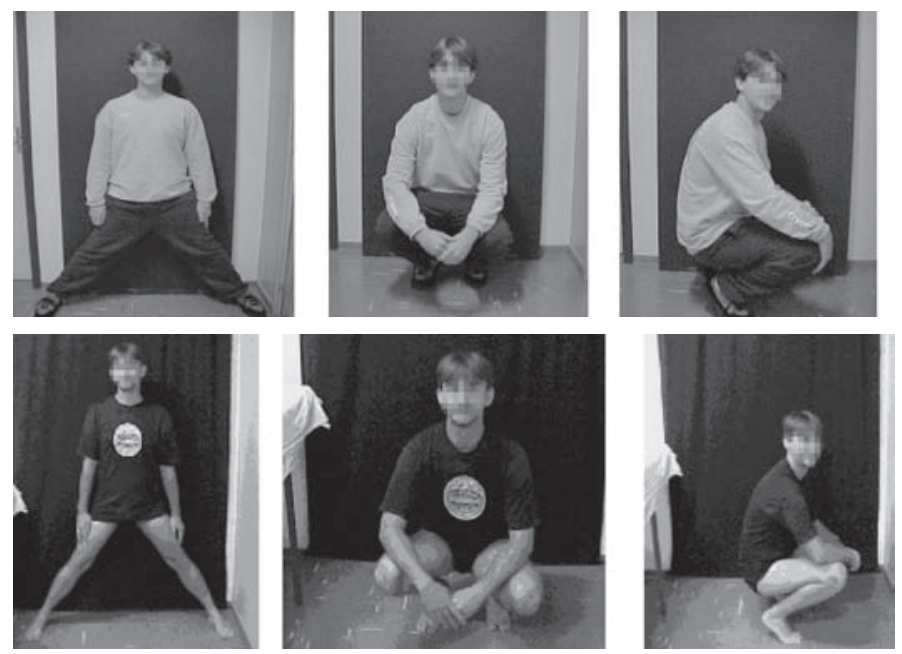

Figura $2 \mathrm{E}$ - Imagens superiores do paciente após a consolidação. Imagens inferiores do paciente após sete anos de seguimento.

Revisando 15 casos concluíram que o momento abdutor e o off-set femoral diminuíram $11 \mathrm{~mm}$ e 21 mm respectivamente quando comparados com o lado normal. Ainda concluíram que o ângulo cervico diafisário aumentou de $123^{\circ}$ para $149^{\circ}$.

As mesmas alterações mecânicas foram encontradas nesta série. Em média o momento abdutor diminui $8,9 \mathrm{~mm}$ que representa percentualmente e teoricamente um decréscimo de 12,1\% de força abdutora.

Entretanto a diferença em relação ao off-set femoral foi muito mais significativa. $O$ decréscimo foi em média 
de 20,6mm. Percentualmente a diminuição do off-set foi de $45,3 \%$. Entretanto mesmo com esta variação importante deve se considerar que a alteração do off-set não tem o mesmo impacto que o momento abdutor na força dos pelvi trocantéricos.

Mathews et $\mathrm{al}^{(5)}$ encontraram claudicação em 13 (86,6\%) de sua série de 15 pacientes e justificou-a por estas alterações biomecânicas. Entretanto este achado não foi constante nesta série, pois apenas oito dos 28 quadris consolidados apresentaram algum tipo de claudicação (28,0\%). Ballmer et $\mathrm{al}^{(4)}$ revendo resultados clínicos de 13 pacientes referem que apenas dois claudicavam (15,4\%) (Figura 3).

Evidentemente, também foram observadas alterações do ângulo cervicodiafisário. A média dos casos alterou de $131,5^{\circ}$ para $144,4^{\circ}$.

Até a última avaliação quatro casos evoluíram para artroplastia do quadril, mas a consolidação foi obtida nos 28 casos restantes $(87,4 \%)$.
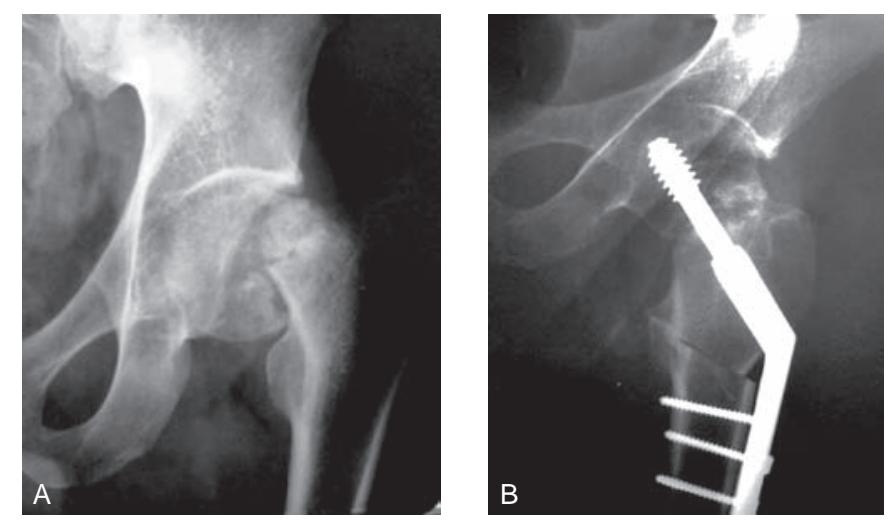

Figura 3 A - N.F, 35 anos, masc.: Rx inicial mostrando pseudartrose do colo femoral. B - pseudartrose e osteotomia consolidada após um ano. Osteossíntese com DHS.
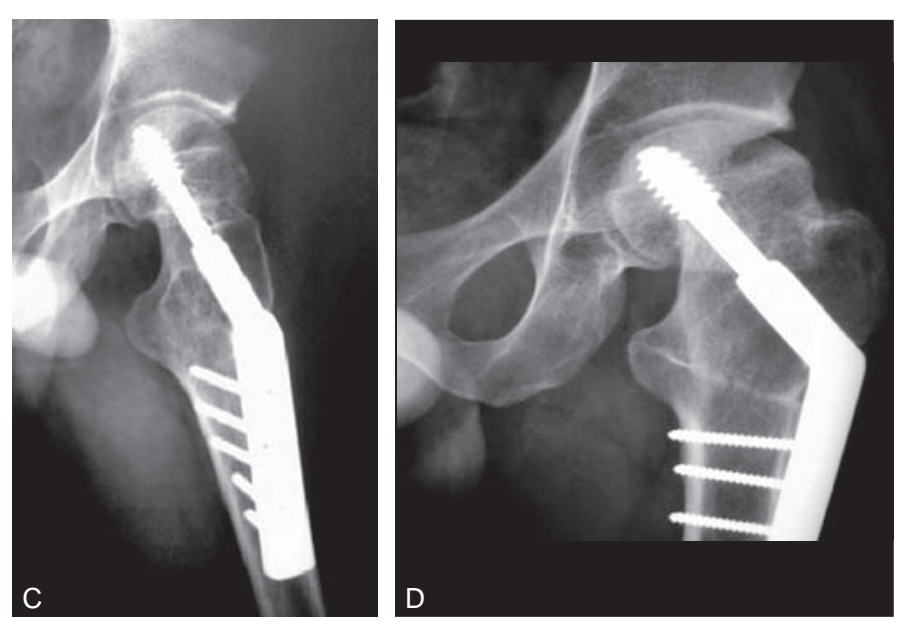

Figura $3 \mathrm{C}$ - N.F. RX após quatro anos e três meses. Observar que o parafuso do DHS alcança o osso subcondral. D - Rx panorâmico da bacia após seguimento de 12 anos.
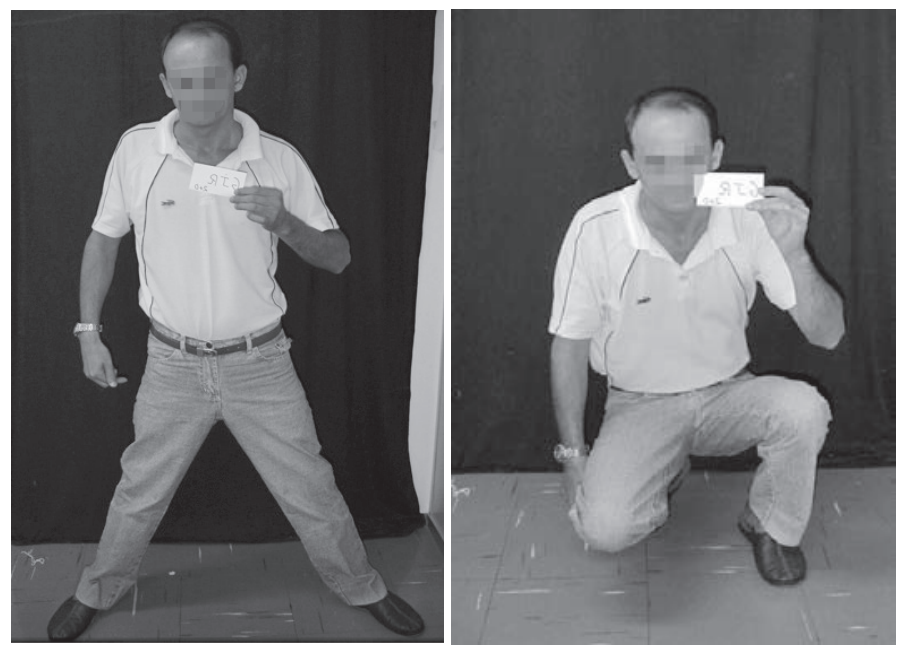

Figura 3 E - Aspecto funcional do paciente após 12 anos.

Pidhorz et $\mathrm{al}^{(20)}$ obtiveram consolidação em $74 \%$, Marti et $\mathrm{al}^{(7)}$ em 86\%, Wentzensen et $\mathrm{al}^{(21)}$ em $100 \%$, Mathews et $\mathrm{al}^{(5)}$ em $80 \%$, Ballmer et $\mathrm{al}^{(4)}$ em $88 \%$ e Wu et $\mathrm{al}^{(22)}$ em $94 \%$.

Apesar do alto índice de consolidação que esta osteotomia alcança é sabido que em muitos casos pode haver necrose, necrose com reossificação e colapso parcial ou total da cabeça femoral. Estas alterações degenerativas tendem a evoluir, mas podemos encontrar resultados clínicos satisfatórios por muitos anos ${ }^{(7)}$. Evidente que se as alterações degenerativas se tornarem progressivas haverá necessidade da artroplastia total do quadril em algum momento do seguimento. Marti et $\mathrm{al}^{(7)}$ com seguimento de 7,1 anos relataram que 14\% evoluíram para a artroplastia, Mathews et $\mathrm{al}^{(5)}$ após quatro anos referiram $15,3 \%$, Ballmer et $\mathrm{al}^{(4)}$ citam dois casos, $11,7 \%$.

Na presente série, oito casos evoluíram com necrose. Somente um caso com 14 anos de evolução foi considerado estágio quatro de Inoue ${ }^{(6)}$, mas ainda apresentava boa mobilidade, dor tolerável e não há previsão da artroplastia total do quadril (Figura 4).

Pela avaliação de D’Aubigne et $\mathrm{al}^{(2)}$, 18 pacientes foram considerados normais com 17 e 18 pontos. Dez obtiveram de 14 a 16 pontos devido dor esporádica, claudicação ou limitação de movimentos.

Revisando a presente série com seguimento médio de 9,8 anos, concluímos que a osteotomia valgizante para o tratamento da pseudartrose do colo do fêmur em pacientes jovens (41,7 anos) foi responsável pela consolidação em 87,4\% (28 casos) pela recuperação integral da função dos quadris em 56,2\% (18 casos). Figuras 2, 3 e 4.

A outra grande alternativa de tratamento para pseudoartrose sem dúvida é a artroplastia total do quadril. 

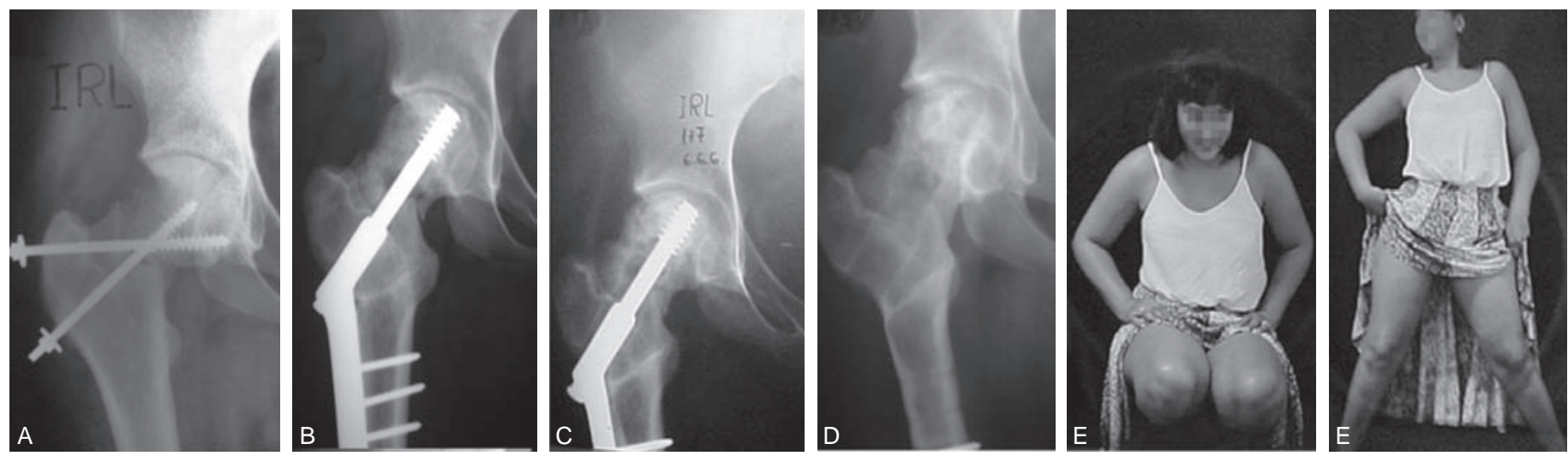

Figura 4 A - G.J.R., 37 anos, masculino, pseudartrose do colo do fêmur. Fratura negligenciada há cinco meses. Observar reabsorção dos bordos da fratura. B - Rx em detalhe após osteossíntese com DHS e osteotomia valgizante. C e D - Imagem radiográfica em detalhe em perfil e AP após a consolidação da osteotomia e pseudartrose. E - Aspecto clínico do paciente após cinco anos.

Ela promove rápido alívio da dor e mobilização precoce. Entretanto resultados a longo prazo nem sempre são satisfatórios sendo encontrados altas taxas de falência e maus resultados em pacientes jovens ${ }^{(23-27)}$. Nestes pacientes a artroplastia total do quadril pode ser a solução mais fácil, porém não é a melhor. Nestes pacientes para tratar uma pseudoartrose do colo do fêmur uma atitude mais conservadora merece ser considerada: A Osteotomia valgizante de Pauwels ${ }^{(1)}$.

\section{REFERÊNCIAS}

1. Pauwels F. Der Schenkelhalsbruch ein mechanisches Problem: Grundlagen des Heilungsvorganges, Prognose und kausale Therapie. Stuttgart: Ferdinand Enke Verlag; 1935.

2. D'Aubigne RM, Postel M. functional results of hip arthroplasty with acrylic prosthesis. J Bone Joint Surg Am. 1954; 36:451-75.

3. Dickson JA. The "unsolved fracture". A protest against defeatism. J Bone Joint Surg Am. 1953; 35(4):805-22.

4. Ballmer FT, Ballmer PM, Baumgaertel F, Ganz R, Mast JW. Pauwels osteotomy for nonunions of the femoral neck. Orthop Clin North Am 1990;21:759-67.

5. Mathews V, Berry D, Trousdale RT, Cabanela M. Clinical and functional results of valgus intertrochanteric osteotomy for femoral neck nonunion. 70th Annual Meeting proceedings AAOS; 2003. p.380.

6. Inoue A, Ono K.: A histological study of idiopathic avascular necrosis of the head of the femur. J Bone Joint Surg Br. 1979;61:138-43.

7. Marti RK, Schuller HM. Raaymakers EL. Intertrochanteric osteotomy for nonunion of the femoral neck. J Bone Joint Surg Br. 1989;71:782-7.

8. Bonfiglio M, Yoke EM. Aseptic necrosis of the femoral head and non-union of the femoral neck: effect of treatment by drilling and bone-grafting (Phemister technique). J Bone Joint Surg Am. 1968;50:48-66.

9. Nagi ON, Dhillon MS, Goni VG. Open reduction, internal fixation and fibular autografting for neglected fracture of the femoral neck. J Bone Joint Surg Br. 1998;80:798-804.

10. Meyers MH, Harvey JP Jr, Moore TM. Treatment of dispraced subcapital and transcervical fractures of the femoral neck by muscle-pedicle-bone graft and internal fixation. A preliminary report on one hundred and fifty cases. J Bone Joint Surg Am. 1973;55:257-74.

11. LeCroy CM, Rizzo M, Gunneson EE, Urbaniak JR. Free vascularized fibular bone grafting in the management of femoral neck nonunion in patients younger than fifty years. J Orthop Trauma. 2002;16:464-72.

12. Protzman RR, Burkhalter WE. Femoral-neck fractures in young adults. J Bone Joint Surg Am. 1976;58:689-95.

13. Swiontkowski MF, Winquist RA, Hansen ST Jr. Fractures of the femoral neck in patients between the ages of twelve and forty-nine years. J Bone Joint Surg Am. 1984;66:837-46.

\section{CONCLUSÕES}

Revisando 32 casos de pseudartrose do colo do fêmur em pacientes jovens (41,7\% anos) concluímos que a alternativa biológica (osteotomia valgizante) foi responsável por alto índice de consolidação 32 casos (87,4\%). Com seguimento médio de 9,8 anos apenas quatro casos $(12,6 \%)$ evoluíram para a artroplastia total do quadril.

14. Barnes R, Brow JT, Garden RS, Nicoll EA. Subcapital fractures of the femur. A prospective review. J Bone Joint Surg Br. 1976;58:2-24.

15. Dedrick DK, Mackenzie JR, Burney RE: Complications of femoral neck fracture in young adults. J Orthop Trauma. 1986;26:932-7.

16. Rajan DT, Parker MJ. Does the level of an intracapsular femoral fracture influence fracture healing after internal fixation? A study of 411 patients. Injury. 2001;32:53-6.

17. Stromqvist B, Hansson LI, Nilsson LT, Thorngren KG. Hook-pin fixation in femoral neck fractures. A two-year follow-up study of 300 cases. Clin Orthop Relat Res. 1987;(218): 58-62.

18. Alho A, Benterud JG, Solovieva S. Internally fixed femoral neck fractures. Early prediction of failure in 203 elderly patients with displaced fractures. Acta Orthop Scand. 1999;70:141-4.

19. Hernefalk L, Messner K. Rigid osteosynthesis decreases the late complication rate after femoral neck fracture: the influence of three different osteosynthesis devices evaluated in 369 patients. Arch Orthop Trauma Surg. 1996;115:71-4.

20. Pidhorz L, Lahbabi S, Deburge A. Les pseudarthroses du col. Femoral: Traitement par osteotomie interthrochanterienne. Rev Chir. Orthop Reparatrice Appar Mot. 1974;60:205-21.

21. Wentzensen A, Weller S. Die Pseudarthrose als komplikation der schenkelhalsfraktur. Aktuelle Traumatol. 1983;13:72-6.

22. Wu CC, Shih $\mathrm{CH}$, Chen WJ, Tai CL. Treatment of femoral neck nonunions with a sliding compression screw: comparison with and without subtrochanteric valgus osteotomy. J Trauma. 1999;46:312-7.

23. Delamarter R, Moreland JR. Treatment of acute femoral neck fractures With total hip arthroplasty. Clin Orthop Relat Res. 1987;(218):68-74.

24. Dorr LD, Glousman R, Hoy AL, Vanis R, Chandler R. Treatment of femoral neck fractures with total hip replacement versus cemented and noncemented hemiarthroplasty .J Arthroplasty. 1986;1:21-8.

25. Franzen H, Nilsson LT, Stromqvist B, Johnsson R, Herrlin K. Secondary total hip replacement after fractures of the femoral neck. J Bone Joint Surg Br. 1990;72:784-7.

26. Greenough CG, Jones JR: Primary total hip replacement for displaced subcapital fractures of the femur. J. Bone Joint Surg Br. 1988;70:639-43.

27. Hunter GA. Should we abandon primary prosthetic replacement for fresh displaced fractures of the neck of the femur? Clin Orthop Relat Res. 1980;(152):158-61. 